\title{
Mixed RF/FSO Relaying With Outdated Channel State Information
}

\author{
Goran T. Djordjevic, Member, IEEE, Milica I. Petkovic, Student Member, IEEE, \\ Aleksandra M. Cvetkovic, Student Member, IEEE, and George K. Karagiannidis, Fellow, IEEE
}

\begin{abstract}
We investigate the outage probability and the average bit error rate (BER) performance of a dual-hop amplify-andforward (AF) relaying system, composed of a mixed radio frequency (RF)/free-space optical (FSO) link, when simultaneously outdated channel state information (CSI) is assumed at the relay and there is a misalignment between transmitter and receiver apertures in FSO link. In contrast to the majority of works on CSI-assisted AF relays, in this paper, we assume that the estimated CSI is outdated, when the relay amplifies the transmitted signal. The RF link experiences Rayleigh fading, while the FSO link is under the influence of atmospheric turbulence, modeled by the Gamma-Gamma distribution. Novel analytical expressions for the outage probability and the average BER are derived in a power series form, which in some special cases are simplified to offer engineering insight into the effects of important transceiver and channel parameters on the system performance. Numerical and simulation results show that there is an optimal value of the transmitter beam waist, which minimizes the overall outage probability. This optimal value strongly depends on the pointing errors standard deviation. Furthermore, the outage probability varies for several orders of magnitude depending on the transmitter beam waist.
\end{abstract}

Index Terms-Bit error rate, fading, free-space optical systems, Gamma-Gamma distribution, outage probability, pointing errors, radio frequency systems, relay.

\section{INTRODUCTION}

$\mathbf{F}$ REE-space optical (FSO) systems have been intensively investigated during last years as an alternative or complement to the radio frequency (RF) systems. The FSO provides many benefits compared to the RF systems, such as licensefree operation, low-cost, simple installation, low power, as well as larger bandwidth supporting more users [1]-[8]. Due to the aforementioned advantages, FSO systems are used for various applications, as the last mile access network, to bridge the

Manuscript received May 28, 2014; revised November 10, 2014; accepted April 10, 2015. Date of publication May 13, 2015; date of current version August 17, 2015. This work was supported by European Science Foundation under COST IC1101 OPTICWISE Action "Optical Wireless Communications-An Emerging Technology." The work of G. T. Djordjevic, M. I. Petkovic and A. M. Cvetkovic was partially supported by Ministry of Education, Science and Technology Development of Republic of Serbia under grant TR-32028, and Ministry of Foreign Affairs of Norway within the NORBAS project.

G. T. Djordjevic, M. I. Petkovic, and A. M. Cvetkovic are with the Faculty of Electronic Engineering, University of Nis, Nis 18000, Serbia (e-mail: goran@elfak.ni.ac.rs; milica.petkovic@elfak.ni.ac.rs; aleksandra. cvetkovic@elfak.ni.ac.rs).

G. K. Karagiannidis is with the Aristotle University of Thessaloniki, Thessaloniki 541 24, Greece, and also with the Khalifa University, Abu Dhabi 127788, UAE (e-mail: geokarag@ auth.gr).

Digital Object Identifier 10.1109/JSAC.2015.2433055 bandwidth gap between the end-users and the fibre optic backbone network. Furthermore, FSO was proved to be an effective solution for disaster recovery, while it is very helpful in difficult terrains [2].

\section{A. Motivation}

In terrestrial FSO systems, the optical signal transmission is affected by path loss, atmospheric turbulence and pointing errors. The path loss is described by deterministic models, while the atmospheric turbulence can be successfully modeled by the Gamma-Gamma distribution, due to its agreement with experimental results in a wide range of turbulence conditions [3], [4]. Log-normal and modified Ricean distributions can be also used for modeling atmospheric turbulence [3], while recently, Málaga distribution was proposed for modeling atmospheric turbulence [9], [10]. One of the substantial effects, which influences the optical signal transmission through free space, is the misalignment between transmitter laser and the photodetector at the receiver. This dynamic misalignment, caused by building sway and vibration, results in pointing errors (jitter or misalignment fading) that affects the FSO link performance, especially when transmitter and receiver apertures are placed on high buildings [1], [11]-[15]. Considering horizontal and vertical displacements (jitters) as independent Gaussian random variables (RVs), with zero mean value and standard deviation denoted by $\sigma_{s}$, the resulting radial displacement at the receiver detector follows Rayleigh distribution [12].

Intensity-modulation/direct detection (IM/DD) with on-off keying $(\mathrm{OOK})$ is a widely employed technique in commercial FSO systems. Although IM/DD with OOK is cost effective and easy to be implemented, the main flaw of OOK is the requirement for adaptive threshold setting for demodulation [2]. As alternative to IM/DD with OOK, the subcarrier intensity modulation (SIM) technique has been proposed to improve the performance of FSO systems. Applying the SIM implies that the RF subcarrier signal is premodulated by the information data sequence, before it is used for modulation of the laser source intensity [2], [16]-[18]. Besides SIM, the FSO systems with coherent detection have been intensively studied, referring to systems where optical wave, generated by local oscillator, is added to the received optical signal [2].

In order to extend the coverage in wireless networks, relaying technology has been accepted in the RF wireless standards [19]. Furthermore, mixed dual-hop amplify-and-forward (AF) relaying systems, consisting of the RF and FSO links, 
were recently investigated in [20]-[26]. The connectivity gap between a backbone and a last-mile access network can be overcome by using the RF/FSO communication, enabling a large number of RF users to be multiplexed through a single FSO link [22]. There are several reasons for selecting FSO technology for the last mile connectivity: The capacity of FSO is comparable with that of the optical fiber, but deployment cost is significantly lower. Moreover, the FSO is transparent regarding several traffic types and protocols and consequently can be easily integrated in the access networks. Because of that, there is both practical and scientific interest to investigate the performance of mixed RF/FSO relaying systems and to identify their major impairments and performance limits.

\section{B. Literature}

Scanning the open literature, the authors in [20]-[24] presented the performance of the asymmetric dual-hop relay transmission with mixed RF/FSO links, employed fixed gain relay. Specifically, the outage performance of this system was presented in [20], where RF and FSO links are modeled by Rayleigh and Gamma-Gamma distribution, respectively. Ansari et al. [21] extended this analysis to other performance metrics, as amount of fading, bit error rate (BER), symbol error rate (SER) and ergodic capacity. Furthermore, the impact of the pointing errors on the overall system performance was investigated in [22], while in [23] an outage analysis was presented, by using general Málaga over the FSO link, instead of GammaGamma fading.

Assuming fixed gain relay, an analysis of the asymmetric mixed RF/FSO dual hop relaying system along with a direct RF uplink, was proposed in [24]. In contrast to the mixed RF/FSO systems with fixed gain relay presented in [20]-[24], variable gain relay was assumed in [25], [26]. This kind of relays uses the instantaneous channel state information (CSI) of the previous RF link in order to determine the gain. Especially, closed-form expressions for the BER and the capacity of mixed RF/FSO variable gain dual-hop transmission system, were derived in [25]. Contrary to [24], where fixed gain relay was assumed, in [26] the performance analysis of the similar system with variable gain relay was presented.

\section{Contribution}

The main purpose of the mixed RF/FSO system is to exploit the FSO benefits, while line-of-sight (LOS) component is not available between source and destination nodes. Hence, RF link is utilized to bridge as a non-LOS link between source and relay nodes, while FSO is used as a solution for the "last mile" problem.

In the aforementioned works, the analysis was limited to fixed or variable gain relaying with perfect CSI. However, when variable gain relay is employed, the relay continuously monitors source-relay link and periodically estimates CSI. Due to temporal variation of the RF channel, estimation error occurs since the estimated CSI (used for adjusting relay gain) is not that at the time of signal transmission. Imperfect CSI indicates that the channel estimation at the relay is outdated, when the relay applies it to amplify transmitted signal. Relaying RF sys- tems, where gain determination is performed by outdated CSI, were analyzed in [27]-[29]. These papers showed that outdated CSI has a considerable effect on the system's performance. To the best of the authors' knowledge, neither the RF/FSO relaying systems with outdated CSI, nor the combined effect of pointing errors over the FSO part, together with outdated CSI over RF part, has been investigated in the past. It is clear that the effect of outdated CSI on the overall system performance should not be neglected in practical mixed RF/FSO relaying systems. Depending on the conditions over the FSO part, the effect of outdated CSI on the overall mixed RF/FSO relaying system performance will be more or less emphasized. From a practical point of view, it is important to investigate the effect of outdated CSI in different propagation conditions over the FSO link.

In this paper, we analyze the performance of a dual-hop $\mathrm{RF} / \mathrm{FSO}$ AF relaying system with outdated CSI. It is assumed that the RF link experiences Rayleigh fading and the received electrical signal at the relay is converted to the optical one for retransmission by SIM. The intensity fluctuations of the received optical signal are described by the combined model of atmospheric path loss, Gamma-Gamma atmospheric turbulence [2]-[4] and pointing errors [1], [11]-[15]. New analytical expressions for the system's outage probability and average BER are derived in the form of a fast convergent power series. In some special cases, these expressions are simplified to more tractable forms, providing a better insight into the effects of different system's parameters on the outage and average BER performance. We also provide some simple formulae for the efficient evaluation of the outage and average BER floors.

The main goal of this work is to derive novel analytical expressions, which can be efficiently used for optimizing the beam waist at the transmitter output, in order to minimize the outage probability, when outdated CSI is assumed at the relay. In addition, the effects of outdated CSI on the overall system performance are investigated for different conditions over FSO link. Numerical results are presented and validated by Monte Carlo simulations.

\section{Structure}

The paper is organized as follows. System model is described in Section II, while Section III contains the outage analysis, which is followed by mathematical derivations given in Appendixes A and B. The average BER analysis is presented in Section IV, with mathematical derivation presented in Appendix C. Numerical results with appropriate comments are given in Section $\mathrm{V}$, and some concluding remarks are presented in Section VI.

\section{System Model}

An asymmetric AF dual-hop system is considered, consisting of three nodes: source, $S$, relay, $R$, and destination, $D$. We assume that there is no direct link between $S$ and $D$. In the first part of transmission, the RF modulated signal is transmitted from $S$ to $R$ node via the RF link. The received signal at the node $R$ in the $k$ th symbol interval is given by

$$
r_{R}=h_{S R} r+n_{S R},
$$


where $r$ is the transmitted symbol from the node $S$, which has an average power normalized to one $\left(\mathrm{E}\left[|r|^{2}\right]=1\right)$, with $\mathrm{E}[\cdot]$ to denote mathematical expectation, $h_{S R}$ is the fading amplitude during the $k$ th symbol interval with $\mathrm{E}\left[\left|h_{S R}\right|^{2}\right]=P_{S}$ and $n_{S R}$ represents the additive white Gaussian noise with zero mean and variance $\sigma_{S R}^{2}=\mathrm{E}\left[\left|n_{S R}\right|^{2}\right]$.

In the second part of transmission, the received signal at node $R$ is amplified by a proper relay gain $G$ and retransmitted to the node $D$ via the FSO link. Amplification at node $R$ is performed based on the estimated CSI, which is assumed to be outdated. Following the same procedure as in [27], the relay gain can be tightly bounded by [30]

$$
G^{2}=\frac{1}{\left|\hat{h}_{S R}\right|^{2}},
$$

where $\hat{h}_{S R}$ is an estimated version of the $h_{S R}$. The noise power of the $S-R$ link is neglected in order the statistical analysis to be more tractable.

Applying SIM the RF signal is converted to an optical one as

$$
r_{\text {opt }}=P_{t}\left(1+m G r_{R}\right),
$$

where $P_{t}$ is the average transmitted optical power and $m$ being the modulation index. Next, we assume $m=1$. After removing dc bias and performing an optical-to-electrical conversion, the demodulated signal at node $D$ during the $k$ th symbol interval, is given by

$$
r_{D}=I_{R D} P_{t} G \eta r_{R}+n_{R D},
$$

where $I_{R D}$ is the fading amplitude over the FSO link during the $k$ th symbol interval, $n_{R D}$ is the additive white Gaussian noise over FSO link with zero mean and variance $\sigma_{R D}^{2}=\mathrm{E}\left[\left|n_{R D}\right|^{2}\right]$ and $\eta$ is an optical-to-electrical conversion coefficient.

Using (1), (2), and (4), the overall signal-to-noise ratio (SNR) at the destination is obtained as

$$
\gamma_{e q}=\frac{\left|I_{R D}\right|^{2} P_{t}^{2} G^{2} \eta^{2}\left|h_{S R}\right|^{2}}{\left|I_{R D}\right|^{2} P_{t}^{2} G^{2} \eta^{2} \sigma_{S R}^{2}+\sigma_{R D}^{2}}=\frac{\gamma_{1} \gamma_{2}}{\gamma_{2}+\hat{\gamma}_{1}},
$$

where

$$
\gamma_{1}=\frac{\left|h_{S R}\right|^{2}}{\sigma_{S R}^{2}}
$$

is the instantaneous SNR of the first RF hop. As stated before, the fading envelope over the RF link is modeled by Rayleigh distribution and the average SNR is

$$
\mu_{1}=\mathrm{E}\left[\gamma_{1}\right]=\frac{\mathrm{E}\left[\left|h_{S R}\right|^{2}\right]}{\sigma_{S R}^{2}}=\frac{P_{s}}{\sigma_{S R}^{2}} .
$$

The estimated version of $\gamma_{1}$ is

$$
\hat{\gamma}_{1}=\frac{\left|\hat{h}_{S R}\right|^{2}}{\sigma_{S R}^{2}} .
$$

The instantaneous electrical SNR over the second FSO link is defined as

$$
\gamma_{2}=\frac{\left|I_{R D}\right|^{2} P_{t}^{2} \eta^{2}}{\sigma_{R D}^{2}}
$$

where $I_{R D}$ is the FSO channel fading coefficient. The average electrical SNR can be determined as

$$
\mu_{2}=\frac{\mathrm{E}\left[I_{R D}\right]^{2} P_{t}^{2} \eta^{2}}{\sigma_{R D}^{2}} .
$$

Since the RF hop experiences Rayleigh fading, $\gamma_{1}$ and $\hat{\gamma}_{1}$ are two exponentially distributed and correlated RVs, with joint probability density function (PDF) given by [31]

$$
f_{\gamma_{1}, \hat{\gamma}_{1}}(x, y)=\frac{1}{(1-\rho) \mu_{1}^{2}} e^{-\frac{x+y}{(1-\rho) \mu_{1}}} I_{0}\left(\frac{2 \sqrt{\rho x y}}{(1-\rho) \mu_{1}}\right),
$$

where $\rho$ is the correlation coefficient, and $I_{v}(\cdot)$ represents the $\nu$-th order modified Bessel function of the first kind [32, (8.406)].

The FSO channel gain consists of three parts: path loss, atmospheric turbulence, and pointing errors, i.e., $I_{R D}=I_{a} I_{l} I_{p}$, where $I_{a}$ is an atmospheric turbulence attenuation modeled by Gamma-Gamma distribution, $I_{l}$ denotes a path loss, and $I_{p}$ denotes a component modeling pointing errors.

The atmospheric path loss, $I_{l}$, is described by the exponential Beers-Lambert law as [12]

$$
I_{l}=\exp (-\sigma L)
$$

where $\sigma$ represents the atmospheric attenuation coefficient and $L$ denotes the FSO link length. The atmospheric turbulence is described by Gamma-Gamma distribution, so the PDF of $I_{a}$ is given by [33, (8)]. The PDF of the attenuation due to pointing error, $I_{p}$, is found as [12, (11)], assuming the radial displacement at the receiver experiences Rayleigh distribution with the jitter variance $\sigma_{s}^{2}$. Finally, the PDF of $I_{R D}$ is found as $[13,(12)]$

$$
\begin{aligned}
f_{I_{R D}}\left(I_{R D}\right) & =\frac{\xi^{2} \alpha \beta}{A_{0} I_{l} \Gamma(\alpha) \Gamma(\beta)} \\
\times G_{1,3}^{3,0}\left(\frac{\alpha \beta}{A_{0} I_{l}} I_{R D} \mid\right. & \left.\xi^{2}-1, \alpha-1, \beta-1\right),
\end{aligned}
$$

where $G_{p, q}^{m, n}(\cdot)$ is the Meijer's $G$-function [32, (9.301)], $\alpha$ and $\beta$ are atmospheric turbulence parameters, while $\xi$ and $A_{0}$ represent the parameters relating pointing errors.

The parameters $\alpha$ and $\beta$ represent the effective numbers of small and large scale cells, respectively, which are related to the atmospheric conditions. Assuming plane wave propagation and zero inner scale, they can be expressed as [2], [3]

$$
\begin{aligned}
& \alpha=\left(\exp \left[0.49 \sigma_{R}^{2}\left(1+1.11 \sigma_{R}^{12 / 5}\right)^{-7 / 6}\right]-1\right)^{-1} \\
& \beta=\left(\exp \left[0.51 \sigma_{R}^{2}\left(1+0.69 \sigma_{R}^{12 / 5}\right)^{-5 / 6}\right]-1\right)^{-1},
\end{aligned}
$$

where $\sigma_{R}^{2}$ is the Rytov variance given by

$$
\sigma_{R}^{2}=1.23 C_{n}^{2} k^{7 / 6} L^{11 / 6} .
$$


In (15), $k=2 \pi / \lambda$ is the wave-number, $\lambda$ is the wavelength, $L$ is the propagation distance, and the refractive index is denoted by $C_{n}^{2}$, which varies from $10^{-17} \mathrm{~m}^{-2 / 3}$ to $10^{-13} \mathrm{~m}^{-2 / 3}$ for weak to strong turbulence, respectively.

The pointing errors parameter $\xi$ is determined as $\xi=$ $w_{L_{e q}} /\left(2 \sigma_{s}\right)$, where $w_{L_{e q}}$ is the equivalent beam waist at the receiver, and $\sigma_{s}$ is the standard deviation of pointing errors (jitter) at the receiver. The parameter $w_{L_{e q}}$ is dependent on the beam waist (radius calculated at $e^{-2}$ ) at distance $L, w_{L}$, as [12]-[15]

$$
w_{L_{e q}}^{2}=\frac{w_{L}^{2} \sqrt{\pi} \operatorname{erf}(v)}{2 v \exp \left(-v^{2}\right)}, \quad v=\frac{\sqrt{\pi} a}{\sqrt{2} w_{L}},
$$

where $a$ is the radius of a circular detector aperture. The parameter $A_{0}$ represents the fraction of the collected power at $L=0$, given by $A_{0}=[\operatorname{erf}(v)]^{2}$, where erf(.) is the error function $[32,(8.250 .1)]$.

The beam waist $w_{L}$ of a Gaussian beam is related to beam waist at transmitter, denoted as $w_{0}$, and to the radius of curvature $F_{0}$ by [15]

$$
w_{L}=w_{0} \sqrt{\left(\Theta_{0}+\Lambda_{0}\right)\left(1+1.63 \sigma_{R}^{12 / 5} \Lambda_{1}\right)},
$$

with $\Theta_{0}=1-L / F_{0}, \Lambda_{0}=2 L /\left(k w_{0}^{2}\right)$ and $\Lambda_{1}=\Lambda_{0} /\left(\Theta_{0}^{2}+\Lambda_{0}^{2}\right)$.

Using (10) and (13), the average electrical SNR of the FSO hop can be written as

$$
\mu_{2}=\frac{P_{t}^{2} \eta^{2}}{\sigma_{R D}^{2}} \kappa^{2} A_{0}^{2} I_{l}^{2}
$$

where $\kappa=\xi^{2} /\left(\xi^{2}+1\right)$.

After some mathematical manipulations, the PDF of $\gamma_{2}$ can be derived as

$$
f_{\gamma_{2}}\left(\gamma_{2}\right)=\frac{\xi^{2}}{2 \Gamma(\alpha) \Gamma(\beta) \gamma_{2}} G_{1,3}^{3,0}\left(\alpha \beta \kappa \sqrt{\frac{\gamma_{2}}{\mu_{2}}} \mid \begin{array}{c}
\xi^{2}+1 \\
\xi^{2}, \alpha, \beta
\end{array}\right) .
$$

Using (19) and utilizing [34, (26)], the cumulative distribution function (CDF) of $\gamma_{2}$ is

$$
F_{\gamma_{2}}(x)=\frac{\xi^{2}}{\Gamma(\alpha) \Gamma(\beta)} G_{2,4}^{3,1}\left(\alpha \beta \kappa \sqrt{\frac{x}{\mu_{2}}} \mid \begin{array}{c}
1, \xi^{2}+1 \\
\xi^{2}, \alpha, \beta, 0
\end{array}\right)
$$

\section{Outage Probability Analysis}

The outage probability represents the probability that the instantaneous end-to-end SNR, $\gamma_{e q}$, falls below a predetermined outage threshold, $\gamma_{t h}$. In this case, the outage probability can be written as

$$
P_{\text {out }}=F_{e q}\left(\gamma_{t h}\right)=\operatorname{Pr}\left(\frac{\gamma_{1} \gamma_{2}}{\gamma_{2}+\hat{\gamma}_{1}}<\gamma_{t h}\right),
$$

where $\operatorname{Pr}(\cdot)$ denotes probability. After some mathematical manipulations, (21) can be expressed as

$$
\begin{aligned}
P_{\text {out }} & =\int_{0}^{\infty} \int_{0}^{\infty} \operatorname{Pr}\left(\frac{\gamma_{1} \gamma_{2}}{\gamma_{2}+\hat{\gamma}_{1}}<\gamma_{\text {th }}\right) f_{\gamma_{1}, \hat{\gamma}_{1}}\left(\gamma_{1}, \hat{\gamma}_{1}\right) d \gamma_{1} d \hat{\gamma}_{1} \\
& =1-\int_{0}^{\infty} \int_{0}^{\infty} \operatorname{Pr}\left(\gamma_{2}>\frac{\gamma_{t h} y}{x}\right) f_{\gamma_{1}, \hat{\gamma}_{1}}\left(x+\gamma_{t h}, y\right) d x d y .
\end{aligned}
$$

The complementary cumulative distribution function (CCDF) of $\gamma_{2}$ is defined as $\bar{F}_{\gamma_{2}}(x)=1-F_{\gamma_{2}}(x)$, where $F_{\gamma_{2}}(x)$ is the CDF of $\gamma_{2}$ given by (20).

Substituting (11) and (20) into (22), and using Appendix A, an analytical expression for the outage probability is derived as

$$
\begin{aligned}
P_{\text {out }}= & 1-e^{-\frac{\gamma_{\text {th }}}{\mu_{1}}} \\
& +\sum_{k=0}^{\infty} \sum_{n=0}^{k}\left(\begin{array}{l}
k \\
n
\end{array}\right) \frac{2^{\alpha+\beta-3} \xi^{2} \rho^{k}(1-\rho)^{n-k+1} \mu_{1}{ }^{n-k}}{k !^{2} \pi \Gamma(\alpha) \Gamma(\beta)} \\
& \times \gamma_{t h}^{k-n} e^{-\frac{\gamma_{t h}}{(1-\rho) \mu_{1}}} G_{3,7}^{6,2}\left(\frac{\alpha^{2} \beta^{2} \kappa^{2} \gamma_{t h}}{16 \mu_{2}} \mid \begin{array}{c}
1,-k, \frac{\xi^{2}+2}{2} \\
\chi_{1}
\end{array}\right),
\end{aligned}
$$

with $\chi_{1}=\frac{\xi^{2}}{2}, \frac{\alpha}{2}, \frac{\alpha+1}{2}, \frac{\beta}{2}, \frac{\beta+1}{2}, 1+n, 0$.

When $\xi \rightarrow \infty$, the pointing errors effect is small and neglectable, so the intensity fluctuations of the received optical signal are assumed to originate only from the Gamma-Gamma atmospheric turbulence. Hence, taking the limit of (23) for $\xi \rightarrow$ $\infty$, the outage probability corresponding to the non-pointing errors system is given by

$$
\begin{aligned}
P_{\text {out }}^{G G}= & 1-e^{-\frac{\gamma_{\text {th }}}{\mu_{1}}} \\
& +\sum_{k=0}^{\infty} \sum_{n=0}^{k}\left(\begin{array}{l}
k \\
n
\end{array}\right) \frac{2^{\alpha+\beta-2} \rho^{k}(1-\rho)^{n-k+1} \mu_{1}{ }^{n-k}}{k^{2} \pi \Gamma(\alpha) \Gamma(\beta)} \\
& \times \gamma_{t h}^{k-n} e^{-\frac{\gamma_{t h}}{(1-\rho) \mu_{1}}} G_{2,6}^{5,2}\left(\frac{\alpha^{2} \beta^{2} \gamma_{t h}}{16 \mu_{2}} \mid \begin{array}{c}
1,-k \\
\chi_{2}
\end{array}\right),
\end{aligned}
$$

with $\chi_{2}=\frac{\alpha}{2}, \frac{\alpha+1}{2}, \frac{\beta}{2}, \frac{\beta+1}{2}, n+1,0$.

Since (23) does not provide direct insights into the outage performance, next we investigate possible simplified forms.

\section{A. High SNR Approximation}

In order to simplify the outage probability expression in (23), higher order terms of series representation of the modified Bessel function of the first kind in (42) can be neglected under certain conditions. Using only the first term, this approximation is valid when the argument of $I_{0}(\cdot)$ is small enough (tends to zero), i.e., at high average SNR per RF link and/or low correlation coefficient. 
If only the first term of summation in (23) is included, i.e., $k=0$, the outage probability can be approximated as

$$
\begin{aligned}
& P_{\text {out }}^{\infty} \approx 1-e^{-\frac{\gamma_{\text {th }}}{\mu_{1}}}+\frac{2^{\alpha+\beta-3} \xi^{2}(1-\rho)}{\pi \Gamma(\alpha) \Gamma(\beta)} \\
& \times e^{-\frac{\gamma_{t h}}{(1-\rho) \mu_{1}}} G_{3,7}^{6,2}\left(\frac{\alpha^{2} \beta^{2} \kappa^{2} \gamma_{t h}}{16 \mu_{2}} \mid \begin{array}{c}
1,0, \frac{\xi^{2}+2}{2} \\
\chi_{3}
\end{array}\right),
\end{aligned}
$$

with $\chi_{3}=\frac{\xi^{2}}{2}, \frac{\alpha}{2}, \frac{\alpha+1}{2}, \frac{\beta}{2}, \frac{\beta+1}{2}, 1,0$.

The FSO channel state has an important role in the accuracy of this approximation. The Meijer's $G$-function is a dominant term in (23) when its argument is large, i.e., at low values of the average electrical SNR per FSO hop or during strong turbulence conditions. In that case, this approximation is not accurate. Hence, a higher number of terms for summation in (23) is required. To conclude, considering only the first term for summation in (23), the approximation is tight with the exact outage probability only at the high average SNR over the $\mathrm{RF}$ link and/or low correlation coefficient, when the average electrical SNR over FSO hop is high enough in weak turbulence conditions.

The outage floor in the range of very high values of the average SNR over the RF link (when $\mu_{1}$ tends to infinity) is obtained directly from (25) as

$$
P_{\text {out } 1}^{\text {floor }} \approx \frac{2^{\alpha+\beta-3} \xi^{2}(1-\rho)}{\pi \Gamma(\alpha) \Gamma(\beta)} G_{3,7}^{6,2}\left(\frac{\alpha^{2} \beta^{2} \kappa^{2} \gamma_{t h}}{16 \mu_{2}} \mid \begin{array}{c}
1,0, \frac{\xi^{2}+2}{2} \\
\chi_{3}
\end{array}\right)
$$

Using this expression, the outage floor can be efficiently calculated with appropriate accuracy under the same conditions as in (25).

In the high electrical SNR region of the FSO link, $\mu_{2}$, the Meijer's $G$-functionin (23) tends to zero[35,(07.34.06.0001.01)]. Therefore, the outage probability for any value of $\mu_{1}$ and high value of $\mu_{2}$ can be derived in a very simple form as

$$
P_{\text {out } 2}^{\text {floor }} \approx 1-e^{-\frac{\gamma_{\text {th }}}{\mu_{1}}}
$$

It can be concluded that the outage performance does not depend on the correlation coefficient, at high values of average electrical SNR per FSO hop. This expression can be utilized for efficient and accurate evaluation of the outage floor, which will be illustrated in the following. Based on (27), it is clear that this floor is determined by the average SNR over the RF link and the specified value of the protection ratio.

\section{B. Two Extreme Correlated Cases}

When $\rho=0$, the gain is evaluated based on the uncorrelated CSI. In that case, the modified Bessel function of the first kind in (41) is equal to one [35, (03.02.03.0013.01)], so an accurate expression for the outage probability can be obtained in a similar way as in (25). Substituting $\rho=0$ into (25)

$$
\begin{aligned}
& P_{\text {out }}^{\rho=0}=1-e^{-\frac{\gamma_{\text {th }}}{\mu_{1}}}+\frac{2^{\alpha+\beta-3} \xi^{2}}{\pi \Gamma(\alpha) \Gamma(\beta)} \\
& \times e^{-\frac{\gamma_{t h}}{\mu_{1}}} G_{3,7}^{6,2}\left(\frac{\alpha^{2} \beta^{2} \kappa^{2} \gamma_{t h}}{16 \mu_{2}} \mid \begin{array}{c}
1, \\
\chi_{3}
\end{array} \frac{\xi^{2}+2}{2}\right) .
\end{aligned}
$$

When $\rho=1$, the gain is evaluated based on the fullycorrelated CSI, i.e., the information used for amplification at the relay will be the same as proper CSI of the RF hop. The approximate outage probability for the considered system when $\rho=1$ is given by

$$
\begin{aligned}
P_{\text {out }}^{\rho=1} \approx 1-e^{-\frac{\gamma_{\text {th }}}{\mu_{1}}} & +\frac{\xi^{2}}{\Gamma(\alpha) \Gamma(\beta)} e^{-\frac{\gamma_{\text {th }}}{\mu_{1}}} \\
& \times G_{2,4}^{3,1}\left(\alpha \beta \kappa \sqrt{\frac{\gamma_{\text {th }}}{\mu_{2}}} \mid \begin{array}{c}
1, \xi^{2}+1 \\
\xi^{2}, \alpha, \beta, 0
\end{array}\right) .
\end{aligned}
$$

For the derivation of (29) see Appendix B.

For the special cases of fully correlated or uncorrelated CSI over RF links, the exact and approximate Eqs. (28) and (29) could be efficiently utilized for the estimation of the outage probability. The accuracy of (28) and (29) will be illustrated in Section V.

\section{Average BER Analysis}

Beside the outage probability, the average BER is another important metric of the system performance. In this Section we derive novel analytical expressions for the average BER of the system when binary phase-shift keying (BPSK), i.e., differential BPSK (DBPSK) [31] is applied over RF link and SIM BPSK, i.e., SIM DBPSK [2] is applied over FSO link.

Using the approach introduced in [36], the average BER of some binary modulations can be derived using [36, (12)]

$$
P_{b}=\frac{q^{p}}{2 \Gamma(p)} \int_{0}^{\infty} e^{-q \gamma} \gamma^{p-1} F_{e q}(\gamma) d \gamma
$$

where the parameters $p$ and $q$ depend on the type of used modulation schemes [21], [37], and $F_{e q}(\gamma)$ is the CDF, having the same form as Eq. (23).

Substituting (23) into (30), and following the derivations presented in Appendix C, the final average BER can be written as

$$
\begin{aligned}
& P_{b}=\frac{1}{2}-\frac{1}{2}\left(\frac{1}{\mu_{1} q}+1\right)^{-p} \\
& +\frac{q^{p}}{2 \Gamma(p)} \sum_{k=0}^{\infty} \sum_{n=0}^{k}\left(\begin{array}{l}
k \\
n
\end{array}\right) \frac{2^{\alpha+\beta-3} \xi^{2} \rho^{k}(1-\rho)^{p+1} \mu_{1} p}{k !^{2} \pi \Gamma(\alpha) \Gamma(\beta)\left(1+q(1-\rho) \mu_{1}\right)^{k-n+p}} \\
& \times G_{4,7}^{6,3}\left(\frac{\alpha^{2} \beta^{2} \kappa^{2}(1-\rho) \mu_{1}}{16 \mu_{2}\left(1+q(1-\rho) \mu_{1}\right)} \mid \begin{array}{c}
1,-k, 1-k+n-p, \frac{\xi^{2}+2}{2} \\
\chi_{1}
\end{array}\right) .
\end{aligned}
$$


When BPSK and SIM BPSK, i.e., DBPSK and SIM DBPSK are employed, the values of the parameters are $(p, q)=(0.5,1)$ and $(p, q)=(1,1)$, respectively.

For the non-pointing errors case, the average BER can be found by substituting (24) into (30), or by taking the limit of (31) for $\xi \rightarrow \infty$. When the intensity fluctuations of the received signal originate only from the Gamma-Gamma atmospheric turbulence, the average BER is found as

$$
\begin{aligned}
& P_{b}^{G G}=\frac{1}{2}-\frac{1}{2}\left(\frac{1}{\mu_{1} q}+1\right)^{-p} \\
& +\frac{q^{p}}{2 \Gamma(p)} \sum_{k=0}^{\infty} \sum_{n=0}^{k}\left(\begin{array}{l}
k \\
n
\end{array}\right) \frac{2^{\alpha+\beta-2} \rho^{k}(1-\rho)^{p+1} \mu_{1} p}{k !^{2} \pi \Gamma(\alpha) \Gamma(\beta)\left(1+q(1-\rho) \mu_{1}\right)^{k-n+p}} \\
& \times G_{3,6}^{5,3}\left(\frac{\alpha^{2} \beta^{2}(1-\rho) \mu_{1}}{16 \mu_{2}\left(1+q(1-\rho) \mu_{1}\right)} \mid \begin{array}{c}
1,-k, 1-k+n-p \\
\chi_{2}
\end{array}\right) .
\end{aligned}
$$

\section{A. High SNR Approximation}

Since the average BER expression does not offer direct insights into the system performance, it can be simplified by neglecting the higher order terms of summation in (31). This approximation is valid under the same conditions as in the outage probability approximation given in (25). After some mathematical manipulations, the approximation has the form

$$
\begin{aligned}
& P_{b}^{\infty} \approx \frac{1}{2}-\frac{1}{2}\left(\frac{1}{q \mu_{1}}+1\right)^{-p} \\
& +\frac{2^{\alpha+\beta-4} \xi^{2}(1-\rho)}{\pi \Gamma(\alpha) \Gamma(\beta) \Gamma(p)}\left(\frac{1}{q \mu_{1}(1-\rho)}+1\right)^{-p} \\
& \times G_{4,7}^{6,3}\left(\frac{\alpha^{2} \beta^{2} \kappa^{2}}{16 \mu_{2} q}\left(\frac{1}{q \mu_{1}(1-\rho)}+1\right)^{-1} \mid \begin{array}{c}
1,0,1-p, \frac{\xi^{2}+2}{2} \\
\chi_{3}
\end{array} .\right.
\end{aligned}
$$

In the high average SNR region of the RF link (when $\mu_{1}$ tends to infinity), the BER floor can be obtained from (33) as

$$
\begin{aligned}
P_{b 1}^{\text {floor }} \approx \frac{2^{\alpha+\beta-4} \xi^{2}(1-\rho)}{\pi \Gamma(\alpha) \Gamma(\beta) \Gamma(p)} & \\
& \times G_{4,7}^{6,3}\left(\frac{\alpha^{2} \beta^{2} \kappa^{2}}{16 \mu_{2} q} \mid \begin{array}{cc}
1, & 0, p, \\
\chi_{3}
\end{array}\right) .
\end{aligned}
$$

When $\mu_{2}$ tends to infinity, the Meijer's $G$-function in (31) tends to zero. Hence, the average BER floor in the range of high values of $\mu_{2}$ for any value of $\mu_{1}$ can be derived as

$$
P_{b 2}^{\text {floor }} \approx \frac{1}{2}-\frac{1}{2}\left(\frac{1}{\mu_{1} q}+1\right)^{-p} .
$$

It is worth to notice that BER floor depends on the average SNR over RF link and modulation format applied. We expect that BER decreases with an increase in the transmitted optical power. However, this decrease is limited by the BER floor, which can be estimated by (35).

\section{B. Two Extreme Correlated Cases}

In the case where the gain determination is performed based on uncorrelated CSI, the average BER can be derived using (28) and (30). Alternatively, this average BER can be obtained by substituting $\rho=0$ and $k=0$ into (31) as

$$
\begin{aligned}
P_{b}^{\rho=0}= & \frac{1}{2}-\frac{1}{2}\left(\frac{1}{\mu_{1} q}+1\right)^{-p}+\frac{2^{\alpha+\beta-4} \xi^{2}}{\pi \Gamma(\alpha) \Gamma(\beta) \Gamma(p)}\left(\frac{1}{q \mu_{1}}+1\right)^{-p} \\
& \times G_{4,7}^{6,3}\left(\frac{\alpha^{2} \beta^{2} \kappa^{2} \mu_{1}}{16 \mu_{2}\left(1+q \mu_{1}\right)} \mid \begin{array}{c}
1,0,1-p, \frac{\xi^{2}+2}{2} \\
\chi_{3}
\end{array}\right) \cdot
\end{aligned}
$$

When the gain is determined based on the fully-correlated CSI, i.e., $\rho=1$, the approximate average BER can be derived by substituting (29) into (30). Following similar procedure as in Appendix C, the average BER approximation can be written as

$$
\begin{aligned}
P_{b}^{\rho=1} & \approx \frac{1}{2}-\frac{1}{2}\left(\frac{1}{\mu_{1} q}+1\right)^{-p}+\frac{2^{\alpha+\beta-4} \xi^{2}}{\pi \Gamma(\alpha) \Gamma(\beta) \Gamma(p)}\left(\frac{1}{q \mu_{1}}+1\right)^{-p} \\
& \times G_{3,6}^{5,2}\left(\frac{\alpha^{2} \beta^{2} \kappa^{2} \mu_{1}}{16 \mu_{2}\left(1+q \mu_{1}\right)} \mid \begin{array}{c}
1,1-p, \frac{\xi^{2}+2}{2}, \frac{\alpha^{2}}{2}, \frac{\alpha^{2}+1}{2}, \frac{\beta^{2}}{2}, \frac{\beta^{2}+1}{2}, 0
\end{array}\right) .
\end{aligned}
$$

When average electrical SNR over FSO link tends to infinity, using [35, (07.34.06.0006.01)] Meijer's $G$-function can be represented in the series form containing Gamma functions.

\section{Numerical Results and Simulations}

This section presents numerical results, obtained by using the analytical expressions derived in the previous sections, together with Monte Carlo simulations. The presented novel analytical expressions for the outage probability and the average BER, given by (23) and (31), can be numerically evaluated using well-known software packages as Mathematica, Matlab or Mapple. The sufficient number of terms for summation in (23) and (31) depends on the values of different parameters, but mostly on the correlation coefficient. For the results presented here, 100 terms in (23) and (31) are sufficient in order to achieve a relative error of $10^{-5}$. Note that the number of required terms is rapidly deceasing, when the value of correlation coefficient is lower.

Temporally correlated Rayleigh RVs can be generated by using an algorithm from [38], while $I_{R D}$ can be obtained as the product of the factors related to the atmospheric turbulence, path loss and pointing errors $\left(I_{R D}=I_{a} I_{l} I_{p}\right)$. The atmospheric turbulence is modeled by the Gamma-Gamma distribution, which is generated by using the expression, $I_{a}=I_{a X} \times I_{a Y}$, where $I_{a X}$ and $I_{a Y}$ are mutually independent RVs, which follow Gamma distribution. These variables can be generated using the algorithms presented in [39], [40]. The RV $I_{p}$ is obtained based on $[12,(9)]$. The path loss is determined by (12). Values of the outage probability and the average BER are estimated using $10^{9}$ generated samples. The numerical results are in agreement with the simulation results. 


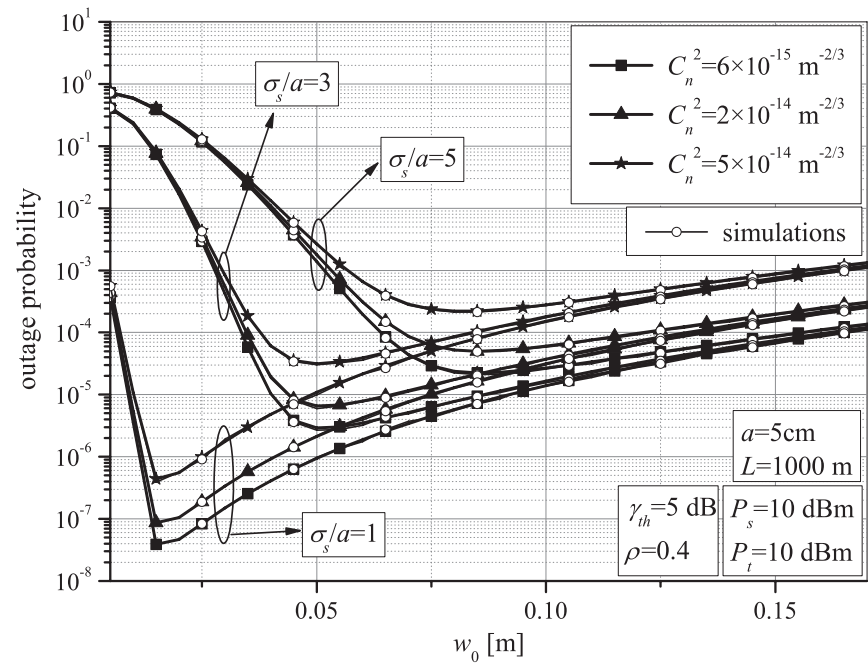

Fig. 1. Outage probability versus transmitter beam waist for different values of normalized jitter standard deviation in various turbulence conditions.

In order to obtain the numerical results for the outage probability and the average BER, the following values of the parameters are used: Turbulence parameters $\alpha$ and $\beta$ are defined by (14), while the turbulence strength is determined by the refractive index parameter as $C_{n}^{2}=6 \times 10^{-15} \mathrm{~m}^{-2 / 3}$ for weak, $C_{n}^{2}=2 \times 10^{-14} \mathrm{~m}^{-2 / 3}$ for moderate and $C_{n}^{2}=5 \times$ $10^{-14} \mathrm{~m}^{-2 / 3}$ for strong turbulence conditions [41]. The wavelength $\lambda=1550 \mathrm{~nm}$ and the optical-to-electrical conversion coefficient, $\eta=1$. The atmospheric attenuation coefficient $\sigma=$ $0.43 \mathrm{~dB} / \mathrm{km}$, which corresponds to clear air conditions [42], [43]. The noise power at the relay is $\sigma_{S R}=-90 \mathrm{dBm}$ [44], while $\sigma_{R D}=10^{-7} \mathrm{~A} / \mathrm{Hz}$ [42], [43]. The radius of a circular detector aperture is $a=5 \mathrm{~cm}$, while the radius of curvature is $F_{0}=-10 \mathrm{~m}[15]$.

Fig. 1 shows the outage probability dependence on the beam waist at the transmitter output, for different values of the normalized jitter standard deviation, when turbulence conditions are changed from weak to heavy ones. As it can be observed, the system performance is better in weak than that in moderate and strong turbulence conditions. Furthermore, the minimum of the outage probability exists for a certain optimal value of $w_{0}$. The system performance can be significantly improved by the proper selecting transmitter aperture. It is clear that the outage probability can vary for several orders of magnitude in dependence on the value of transmitter output beam waist for all turbulence conditions. From the results depicted in Fig. 1 we can conclude that the negative mismatch of the transmitter beam waist from the optimal has a detrimental effect on the outage probability.

The optimal values of the transmitter beam waist, $w_{0_{\text {opt }}}$, assuming the system parameters from Fig. 1, for different values of the normalized jitter standard deviation in various turbulence conditions, are given in Table I. It can be noticed that the normalized jitter standard deviation has strong impact on $w_{0_{\text {opt }}}$. Also, the optimal values of transmitter output beam waist do not depend on the turbulence conditions, when the normalized jitter standard deviation is $\sigma_{s} / a=1$. On the other hand, when $\sigma_{s} / a=5$, reduction of $w_{0}$ ipt noticed, with degradation of the turbulence conditions. The jitter standard deviation is determined by the degree of the misalignment between the FSO transmitter and the receiver apertures. When $\sigma_{s} / a$ is lower, i.e., the parameter $\xi$ is greater, the pointing errors can be neglected. With greater values of $\sigma_{s} / a$, the pointing errors has a dominant effect on the system performance.

Table II presents the calculated values of $w_{0 \text { opt }}$ in moderate turbulence conditions, assuming the system parameters from Fig. 2. Considering values of $w_{0_{\text {opt }}}$ given in Table II, the outage probability dependence on the average transmitted optical power for different values of $\sigma_{s} / a$, is presented in Fig. 2 . With higher average optical power the system has a better performance. Also, decreasing of $\sigma_{S} / a$ leads to an improvement of the outage performance. The results in Fig. 2 illustrate that the effect of the normalized jitter standard deviation on the outage probability is very strong. For example, by changing normalized standard deviation of the radial displacement from $\sigma_{s} / a=1$ to $\sigma_{s} / a=2$, the average transmitted optical power penalty of about $6 \mathrm{dBm}$ should be paid for achieving outage probability of $10^{-5}$. Furthermore, Table II shows that an increase on the average transmitted optical power has no influence on the optimal choice of $w_{0}$, when $\sigma_{s} / a=1$. With higher values of $\sigma_{s} / a$, the pointing errors effect is stronger and the average transmitted optical power has an important role in the determination of $w_{0}$ opt

The optimal transmitter beam waist exists because of the following: Increasing the transmitter beam waist leads to decreasing the effect of pointing errors, but at the same time the received optical power decreases. On the other hand, decreasing transmitter beam waist causes decreasing the geometric loss, but also increasing the effects of pointing errors. From the results presented here, it is evident that significant gains can be achieved in practical mixed RF/FSO systems by accurate selecting the transmitter beam waist.

The outage probability dependence on the average electrical SNR over FSO link for different values of correlation coefficient and normalized jitter standard deviation is presented in Fig. 3. As it was concluded from previous results, the system performance is better when $\sigma_{s} / a$ is lower. The higher value of $\rho$ results in a lower value of the outage probability. The improvement of the system performance, when $\rho$ increases is more evident in the non-pointing error system (lower values of $\left.\sigma_{s} / a\right)$. When $\sigma_{s} / a=5$, the correlation has a weak influence on the system performance. This case corresponds to the great misalignment between FSO transmitter and receiver, when the optical signal is degraded to a large extent, so the correlation is of a less importance on the system performance. Next, Fig. 3 also presents the results obtained by (24), corresponding to the system, when Gamma-Gamma atmospheric turbulence is assumed for the FSO link. The agreement between these results and those for $\sigma_{s} / a=1$ is noticed, which justifies that low values of $\sigma_{s} / a$, i.e., great values of $\xi$, lead to a negligible misalignment between FSO apertures. Furthermore, the existence of a certain outage floor exists at the high values of $\mu_{2}$, which is confirmed by the approximation at high values of $\mu_{2}$ obtained by (27). This floor is dependent only on the average SNR over RF link, so all presented curves tend to the same saturation floor. Although this is not visible in Fig. 3, it would 
TABLE I

Optimum Values of Optical Beam Waist at Transmitter and Receiver

\begin{tabular}{||c||c|c||c|c||c|c||}
\hline \hline \multicolumn{2}{||c||}{$\sigma_{s} / a$} & \multicolumn{2}{c||}{$C_{n}^{2}=6 \times 10^{-15} \mathrm{~m}^{-2 / 3}$} & \multicolumn{2}{c||}{$C_{n}^{2}=2 \times 10^{-14} \mathrm{~m}^{-2 / 3}$} & \multicolumn{2}{c||}{$C_{n}^{2}=5 \times 10^{-14} \mathrm{~m}^{-2 / 3}$} \\
\cline { 2 - 7 } & $w_{0_{\text {opt }}}^{2}[\mathrm{~cm}]$ & $w_{L} / a$ & $w_{0_{\text {opt }}}[\mathrm{cm}]$ & $w_{L} / a$ & $w_{0_{\text {opt }}}[\mathrm{cm}]$ & $w_{L} / a$ \\
\hline \hline 1 & 1.6 & 3.25 & 1.6 & 3.25 & 1.6 & 3.25 \\
2 & 3.4 & 6.85 & 3.4 & 6.85 & 3.3 & 6.65 \\
3 & 5.1 & 10.26 & 5.1 & 10.26 & 5.0 & 10.06 \\
4 & 6.9 & 13.87 & 6.8 & 13.68 & 6.6 & 13.27 \\
5 & 8.6 & 17.29 & 8.5 & 17.1 & 8.2 & 16.49 \\
\hline \hline
\end{tabular}

TABLE II

Optimum Values of Optical Beam Waist at Transmitter OUtPut

\begin{tabular}{||c||c|c|c|c|c||}
\hline \hline \multirow{2}{*}{$P_{t}[\mathrm{dBm}]$} & \multicolumn{5}{|c||}{$w_{0_{\text {opt }}}[\mathrm{cm}]$} \\
\cline { 2 - 6 } & $\sigma_{s} / a=1$ & $\sigma_{s} / a=2$ & $\sigma_{s} / a=3$ & $\sigma_{s} / a=4$ & $\sigma_{s} / a=5$ \\
\hline \hline-10 & 1.6 & 3.1 & 4.2 & 5.2 & 5.9 \\
-6 & 1.6 & 3.2 & 4.7 & 5.9 & 7.0 \\
-2 & 1.6 & 3.3 & 4.9 & 6.4 & 7.7 \\
2 & 1.6 & 3.4 & 5.0 & 6.7 & 8.2 \\
6 & 1.6 & 3.4 & 5.1 & 6.8 & 8.4 \\
10 & 1.6 & 3.4 & 5.1 & 6.8 & 8.5 \\
\hline \hline
\end{tabular}

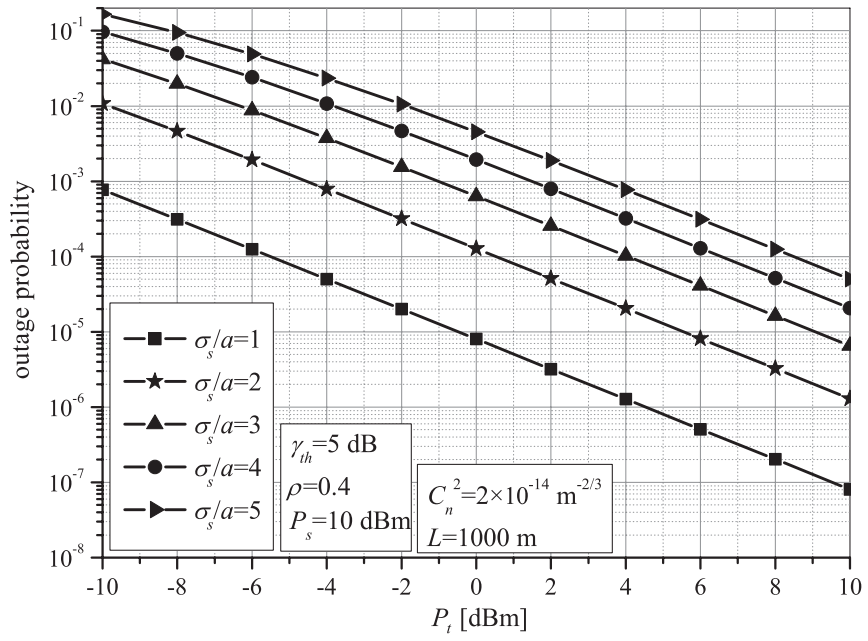

Fig. 2. Outage probability versus average transmitted optical power for different values of normalized jitter standard deviation.

be confirmed by increasing the range of the average electrical SNR, $\mu_{2}$.

Fig. 4 shows the exact outage probability together with corresponding approximation. It is evident that the approximation results obtained by (25) are in a very good agreement with the exact ones, when $\mu_{2}=35 \mathrm{~dB}$. However, when $\mu_{2}=20 \mathrm{~dB}$, the approximation is not accurate, when the correlation coefficient has a relatively great value $(\rho=0.8)$, but it is still in agreement with the exact outage probability, when $\rho$ takes lower values $(\rho=0.2)$. Furthermore, it is clear that the outage floor appears at high values of average SNR per RF link. It can be noticed here that when $\rho$ takes smaller values, the outage floor appears at lower values of $\mu_{1}$.

The outage probability versus average SNR per hops $\left(\mu_{1}=\right.$ $\mu_{2}$ ) for two extreme correlated cases and different values of outage threshold, is presented in Fig. 5. As it is expected, system has a better performance, when the value of $\gamma_{t h}$ is lower. This means that both average SNRs simultaneously increase,

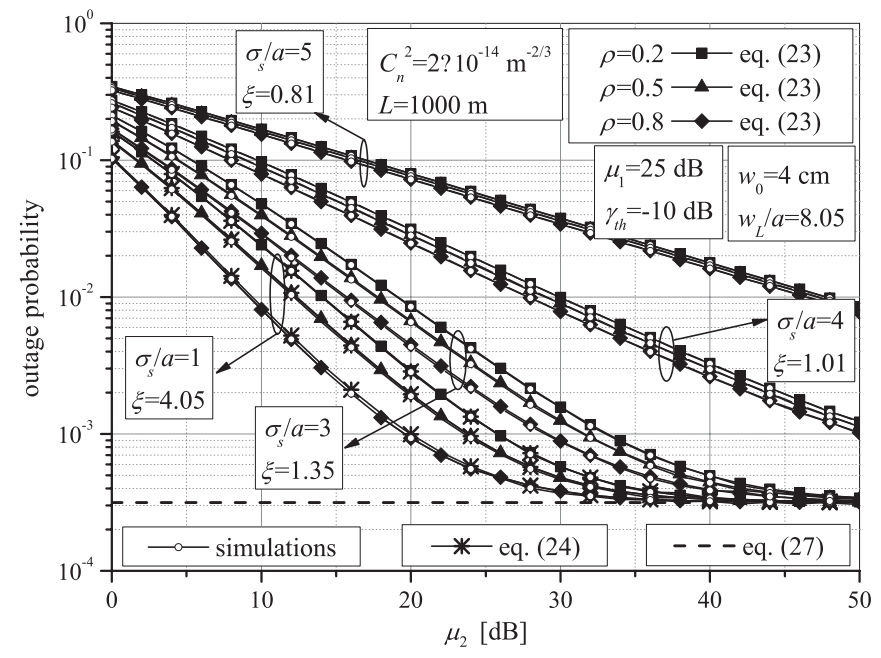

Fig. 3. Outage probability versus $\mu_{2}$ for different values of correlation coefficient and normalized jitter standard deviation.

and neither $\mu_{1}$ nor $\mu_{2}$ is fixed, while one of them increases. This figure also presents the results for uncorrelated and fullycorrelated systems obtained by Eqs. (28) and (29), respectively. Since results for $\rho=1$, i.e., Eq. (29), are achieved by the aforementioned approximation, there is a slight disagreement at lower values of SNRs between this approximation and simulations. If the specified value of outage probability is $10^{-4}$ and $\gamma_{\text {th }}=-10 \mathrm{~dB}$, the required average SNR is $38.6 \mathrm{~dB}$ when $\rho=$ 0 and $30.3 \mathrm{~dB}$ when $\rho=1$. Also, results of the approximation given by (25) are presented.

The average BER performance of the system employing BPSK and BDPSK schemes for different values of normalized jitter standard deviation is presented in Fig. 6. The analytical results obtained by (31) are validated by Monte Carlo simulations. Also, the results for non-pointing errors in the FSO part of the system, obtained by (32), are presented, which are in agreement with the results obtained by (31), for $\sigma_{s} / a=1$. It is noticed that the BER floor occurs at high values of average 


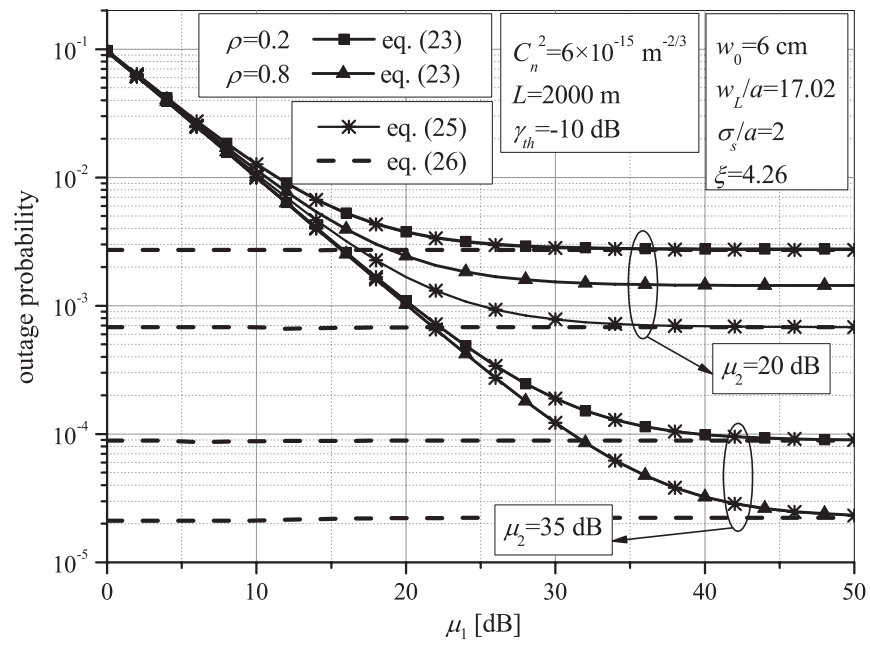

Fig. 4. Outage probability versus $\mu_{1}$ for different values of correlation coefficient and average electrical SNR over FSO link.

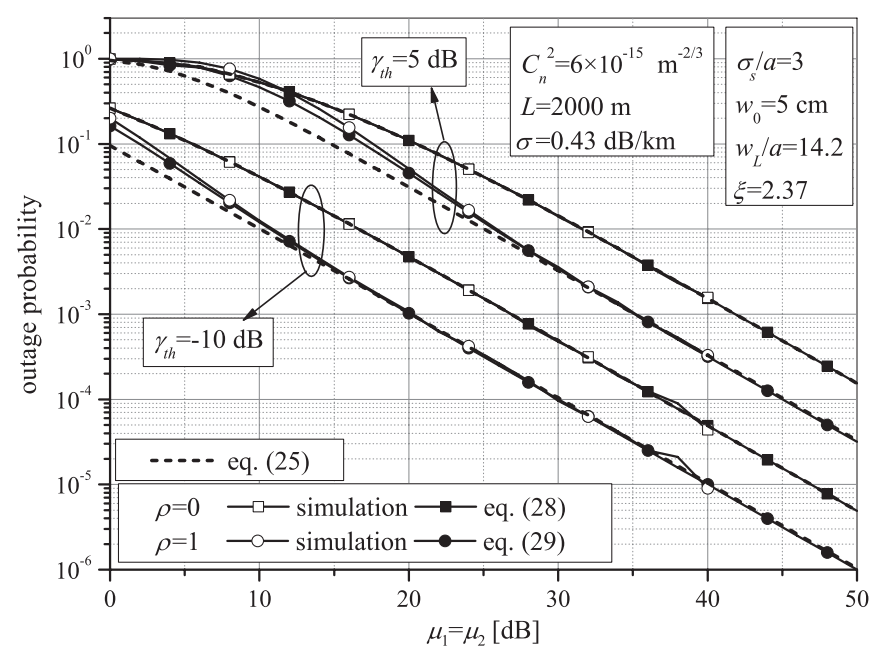

Fig. 5. Outage probability versus $\mu_{1}=\mu_{2}$ for different values of correlation coefficient and outage threshold.

electrical SNR over FSO link. This floor is presented and calculated by (35). As it was concluded in Section IV, the values of BER floor are different for two modulation formats considered here.

Fig. 7 shows the BER dependence on the average SNR per hops $\left(\mu_{1}=\mu_{2}\right)$ for the two extreme correlated cases and different FSO propagation distances. Since the results for the case of $\rho=1$ are obtained by the approximation given in (37), minor disagreement is noticed compared to exact values. Since these disagreement is observed in very low values of SNRs, this range is not significant for the practical implementation and approximation can be successfully applied in the range of SNRs of practical interest. As it is expected, system has worse performance, when the propagation distance between FSO transmitter and receiver is higher. Also, the effect of correlation is more pronounced in the case of $L=1000 \mathrm{~m}$. With a higher length of the FSO hop, the optical signal is more damaged, so the correlation has less effect on the system performance.

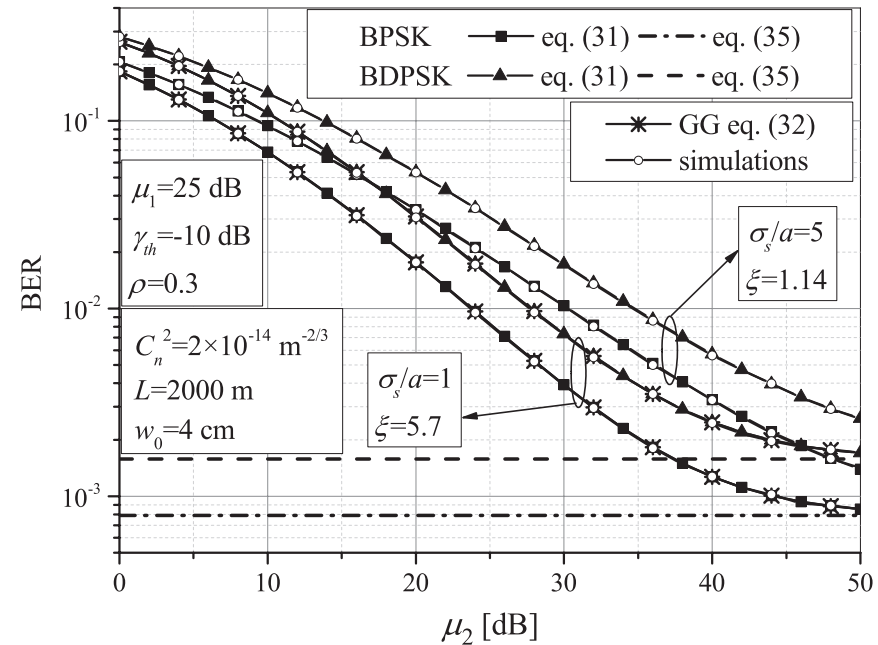

Fig. 6. BER versus $\mu_{2}$ for different values of normalized jitter standard deviation and different types of binary modulations.

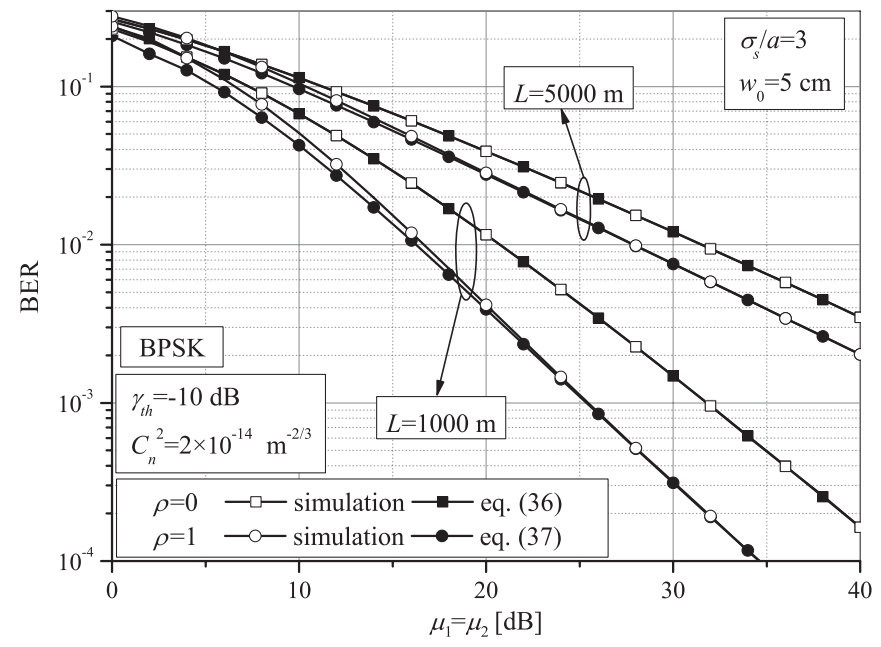

Fig. 7. BER versus $\mu_{1}=\mu_{2}$ for different values of correlation coefficient and propagation distance.

Fig. 8 presents the BER performance of the system with BDPSK for different values of correlation coefficient in weak and strong turbulence conditions. Besides the simulations, the approximation given by (33) is also presented. There is disagreement between these results when $C_{n}^{2}=5 \times 10^{-14} \mathrm{~m}^{-2 / 3}$, since the approximation is valid only in weak turbulence conditions. Based on simulations, it can be concluded that the influence of correlation (i.e., the conditions of the RF system part), is more dominant in weak turbulence. The outage floor, obtained by (34), is also presented. It can be noticed that approximation (34) is valid only under previously defined conditions.

Fig. 9 presents outage and BER floors by using simple relations (27) and (35). Signal transmission in mixed RF/FSO relaying system is influenced by conditions over both RF and FSO channel. As it has been illustrated in Fig. 3, the outage probability decreases with an increase in the average electrical SNR over FSO channel, while the average SNR over the RF channel is fixed. This decrease is expressed in the range of low and moderate values of the average electrical SNR over 


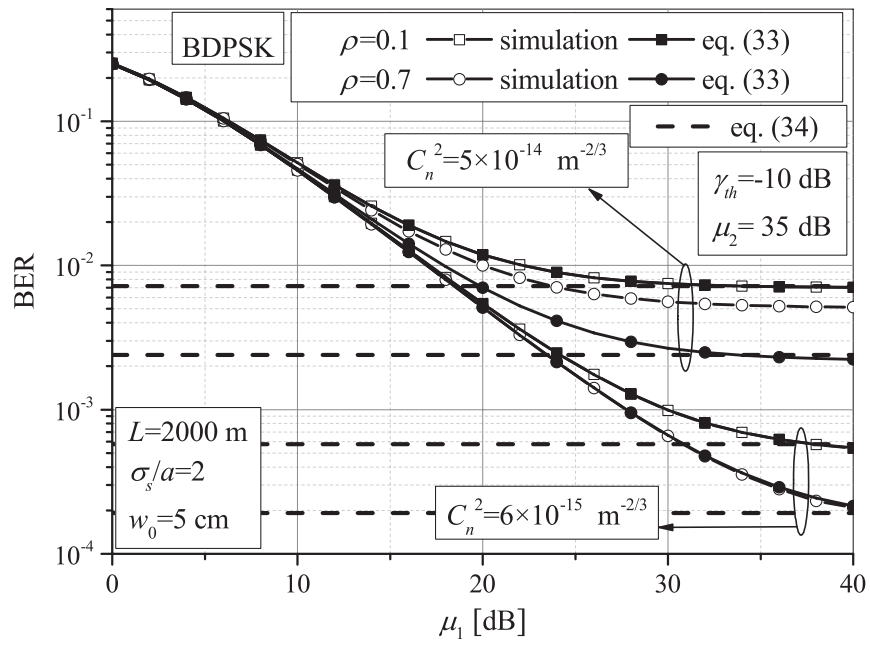

Fig. 8. BER versus $\mu_{1}$ for different values of correlation coefficient in different turbulence conditions.

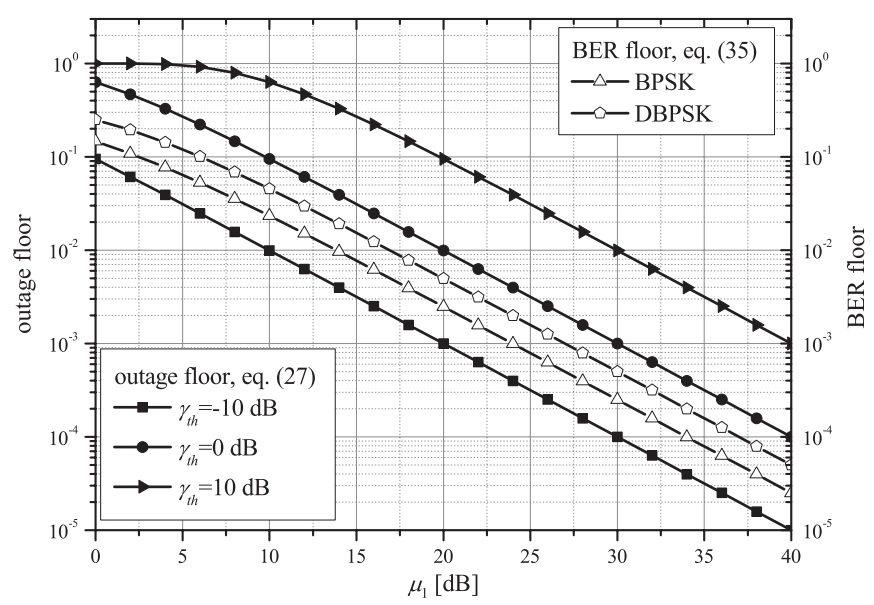

Fig. 9. Outage and BER floors.

the FSO channel. For high values of the average electrical SNR over FSO, the outage probability tends to constant value regardless further increasing the average electrical SNR over the FSO channel. This outage floor can not be decreased by improving the conditions over FSO channel, but is determined by conditions over RF channel. The quite similar behavior in the case of BER is illustrated in Fig. 6. In other words, plots in Fig. 9, obtained on the basis of (27) and (35) illustrate what the best overall performance could be achieved under fixed conditions over RF channel.

\section{CONCLUSION}

In this paper, we have derived novel analytical expressions for the outage probability and the average BER of a mixed dual-hop RF/FSO relaying system with AF relaying, assuming outdated channel estimation in relay gain determination, as well as the presence of the misalignment fading over the FSO link. The analytical results have been confirmed via Monte Carlo simulations. Furthermore, simpler expressions for the outage probability and the average BER have been derived and utilized for the efficient evaluation of the system performance, in special cases of uncorrelated or fully-correlated CSI, as well as for the estimation of the outage and BER floor. The effects of turbulence strength, pointing errors, signal power and correlation coefficient on the outage probability and the average BER have been also discussed.

Based on the derived analytical results, it was proved that there is an optimal value of the transmitter beam waist, which minimizes the outage probability. The greater the value of normalized jitter standard deviation, the stronger are the effects of turbulence strength and average transmitting optical power on the optimal transmitter beam waist. The outage probability can increase even for several orders of magnitude depending on value of transmitter beam waist. The results have illustrated the performance degradation of about $6 \mathrm{dBm}$ at target outage probability of $10^{-5}$, when normalized jitter standard deviation changes from 1 to 2 . We have found that the impact of the outdated CSI on the overall system performance is stronger if FSO propagation distance is shorter and in weaker turbulence conditions, as well as when jitter standard deviation is lower.

\section{APPENDIX A}

\section{DERIVATION OF EQ. (23)}

Eq. (22) can be reproduced here as

$$
\begin{aligned}
P_{\text {out }} & =1-\int_{0}^{\infty} \int_{0}^{\infty} \frac{1}{(1-\rho) \mu_{1}^{2}} e^{-\frac{x+\gamma_{\text {th }}+y}{(1-\rho) \mu_{1}}} I_{0}\left(\frac{2 \sqrt{\rho\left(x+\gamma_{\text {th }}\right) y}}{(1-\rho) \mu_{1}}\right) \\
& \times\left(1-\frac{\xi^{2}}{\Gamma(\alpha) \Gamma(\beta)} G_{2,4}^{3,1}\left(\alpha \beta \kappa \sqrt{\frac{\gamma_{t h} y}{\mu_{2} x}} \mid \begin{array}{c}
1, \xi^{2}+1 \\
\xi^{2}, \alpha, \beta, 0
\end{array}\right)\right) d x d y,
\end{aligned}
$$

and, after some manipulations,

$$
P_{\text {out }}=1-I_{1}+I_{2} \text {, }
$$

where integrals $I_{1}$ and $I_{2}$ are defined and solved in the following.

Using [32, (6.614.3)], [35, (07.44.03.0007.01)] and [32, (2.311)]

$$
I_{1}=\int_{0}^{\infty} \int_{0}^{\infty} \frac{e^{-\frac{x+\gamma_{t h}+y}{(1-\rho) \mu_{1}}}}{(1-\rho) \mu_{1}^{2}} I_{0}\left(\frac{2 \sqrt{\rho\left(x+\gamma_{t h}\right) y}}{(1-\rho) \mu_{1}}\right) d x d y=e^{-\frac{\gamma_{t h}}{\mu_{1}}}
$$

The second integral, $I_{2}$, is

$$
\begin{gathered}
I_{2}=\frac{\xi^{2} e^{-\frac{\gamma_{t h}}{(1-\rho) \mu_{1}}}}{\Gamma(\alpha) \Gamma(\beta)(1-\rho) \mu_{1}^{2}} \int_{0}^{\infty} \int_{0}^{-\frac{x+y}{(1-\rho) \mu_{1}}} I_{0}\left(\frac{2 \sqrt{\rho\left(x+\gamma_{t h}\right) y}}{(1-\rho) \mu_{1}}\right) \\
\quad \times G_{2,4}^{3,1}\left(\alpha \beta \kappa \sqrt{\frac{\gamma_{t h} y}{\mu_{2} x}} \mid \begin{array}{cc}
1, & \xi^{2}+1 \\
\xi^{2}, & \alpha, \beta, 0
\end{array}\right) d x d y
\end{gathered}
$$


where we use [35, (03.02.06.0037.01)] to present the modified Bessel function of the first kind into a series form. Afterwards, using [35, (06.10.03.0018.01)], $I_{2}$ can be written as

$$
\begin{array}{r}
I_{2}=\frac{\xi^{2} e^{-\frac{\gamma_{t h}}{(1-\rho) \mu_{1}}}}{\Gamma(\alpha) \Gamma(\beta)(1-\rho) \mu_{1}^{2}} \int_{0}^{\infty} \int_{0}^{\infty} e^{-\frac{x+y}{(1-\rho) \mu_{1}}} \sum_{k=0}^{\infty} \frac{\rho^{k}\left(x+\gamma_{t h}\right)^{k} y^{k}}{k !^{2}(1-\rho)^{2 k} \mu_{1}^{2 k}} \\
\times G_{2,4}^{3,1}\left(\alpha \beta \kappa \sqrt{\frac{\gamma_{t h y} y}{\mu_{2} x}} \mid \begin{array}{c}
1, \\
\xi^{2}+1 \\
\xi^{2}, \alpha, \beta, 0
\end{array}\right) d x d y, \quad(42)
\end{array}
$$

or

$$
\begin{aligned}
I_{2}=\sum_{k=0}^{\infty} \frac{\xi^{2} \rho^{k} e^{-\frac{\gamma_{t h}}{(1-\rho) \mu_{1}}}}{k !^{2} \Gamma(\alpha) \Gamma(\beta)(1-\rho)^{2 k+1} \mu_{1}^{2 k+2}} \\
\quad \times \int_{0}^{\infty}\left(x+\gamma_{t h}\right)^{k} e^{-\frac{x}{(1-\rho) \mu_{1}}} d x \times I_{21},
\end{aligned}
$$

where

$$
I_{21}=\int_{0}^{\infty} y^{k} e^{-\frac{y}{(1-\rho) \mu_{1}}} G_{2,4}^{3,1}\left(\alpha \beta \kappa \sqrt{\frac{\gamma_{t h y} y}{\mu_{2} x}} \mid \begin{array}{c}
1, \xi^{2}+1 \\
\xi^{2}, \alpha, \beta, 0
\end{array}\right) d y .
$$

In order to solve integral $I_{21}$ it is necessary to represent exponential function in terms of Meijer's $G$-function using [35, (01.03.26.0004.01)]. Afterwards we use [35, (07.34.21.0013.01)] to solve the integral, which involves the product of two Meijer's $G$-functions in the form

$$
\begin{aligned}
I_{21}=\frac{2^{\alpha+\beta-3}(1-\rho)^{k+1} \mu_{1}{ }^{k+1}}{\pi} & \\
& \quad \times G_{5,8}^{6,3}\left(\frac{\alpha^{2} \beta^{2} \kappa^{2} \gamma_{t h}(1-\rho) \mu_{1}}{16 \mu_{2} x} \mid \begin{array}{l}
\chi_{4} \\
\chi_{5}
\end{array}\right),
\end{aligned}
$$

where $\chi_{4}=\frac{1}{2}, 1,-k, \frac{\xi^{2}+1}{2}, \frac{\xi^{2}+2}{2}$ and $\chi_{5}=\frac{\xi^{2}}{2}, \frac{\xi^{2}+1}{2}, \frac{\alpha}{2}, \frac{\alpha+1}{2}$, $\frac{\beta}{2}, \frac{\beta+1}{2}, 0, \frac{1}{2}$.

Substituting (45) into (43), $I_{2}$ can be written as

$$
I_{2}=\sum_{k=0}^{\infty} \frac{2^{\alpha+\beta-3} \xi^{2} \rho^{k} e^{-\frac{\gamma_{t h}}{(1-\rho) \mu_{1}}}}{k !^{2} \pi \Gamma(\alpha) \Gamma(\beta)(1-\rho)^{k} \mu_{1}^{k+1}} \times I_{22},
$$

where

$$
\begin{aligned}
I_{22}=\int_{0}^{\infty}\left(x+\gamma_{t h}\right)^{k} e^{-\frac{x}{(1-\rho) \mu_{1}}} & \\
& \times G_{5,8}^{6,3}\left(\frac{\alpha^{2} \beta^{2} \kappa^{2} \gamma_{t h}(1-\rho) \mu_{1}}{16 \mu_{2} x} \mid \begin{array}{l}
\chi_{4} \\
\chi_{5}
\end{array}\right) d x .
\end{aligned}
$$

After applying binomial theorem [32, (1.111)] and $[35,(07.34 .16 .0002 .01)]$, the integral $I_{22}$ has the form

$$
\begin{aligned}
I_{22}=\sum_{n=0}^{k}\left(\begin{array}{l}
k \\
n
\end{array}\right) \gamma_{t h} h^{k-n} \int_{0}^{\infty} x^{n} e^{-\frac{x}{(1-\rho) \mu_{1}}} & \\
& \times G_{8,5}^{3,6}\left(\frac{16 \mu_{2} x}{\alpha^{2} \beta^{2} \kappa^{2} \gamma_{t h}(1-\rho) \mu_{1}} \mid \begin{array}{l}
\chi_{6} \\
\chi_{7}
\end{array}\right) d x,
\end{aligned}
$$

with $\chi_{6}=1-\frac{\xi^{2}}{2}, 1-\frac{\xi^{2}+1}{2}, 1-\frac{\alpha}{2}, 1-\frac{\alpha+1}{2}, 1-\frac{\beta}{2}, 1-\frac{\beta+1}{2}$, $1, \frac{1}{2}$ and $\chi_{7}=\frac{1}{2}, 0,1+k, 1-\frac{\xi^{2}+1}{2}, 1-\frac{\xi^{2}+2}{2}$.

Using [35, (01.03.26.0004.01)] to present exponential function in terms of Meijer's $G$-function and [35, (07.34.21.0011.01)] to solve an integral of a product of two Meijer's $G$-functions, the integral $I_{22}$ is derived as

$$
\begin{aligned}
& I_{22}=\sum_{n=0}^{k}\left(\begin{array}{l}
k \\
n
\end{array}\right) \gamma_{t h}{ }^{k-n}(1-\rho)^{n+1} \mu_{1}^{n+1} \\
& \times G_{9,5}^{3,7}\left(\frac{16 \mu_{2}(1-\rho) \mu_{1}}{\alpha^{2} \beta^{2} \kappa^{2} \gamma_{t h}(1-\rho) \mu_{1}} \mid \begin{array}{l}
\chi_{8} \\
\chi_{7}
\end{array}\right) d x,
\end{aligned}
$$

with $\chi_{8}=\frac{2-\xi^{2}}{2}, \frac{1-\xi^{2}}{2}, \frac{2-\alpha}{2}, \frac{1-\alpha}{2}, \frac{2-\beta}{2}, \frac{1-\beta}{2},-n, 1, \frac{1}{2}$.

Using [35, (07.34.16.0002.01)], [35, (07.34.03.0001.01)], [35, (07.34.04.0003.01)], [35, (07.34.04.0004.01)] and [35, (07.34.03.0002.01)], the integral $I_{22}$ can be expressed in the form

$$
\begin{aligned}
I_{22}=\sum_{n=0}^{k}\left(\begin{array}{l}
k \\
n
\end{array}\right) & \gamma_{t h}^{k-n}(1-\rho)^{n+1} \mu_{1}^{n+1} \\
& \quad \times G_{3,7}^{6,2}\left(\frac{\alpha^{2} \beta^{2} \kappa^{2} \gamma_{t h}}{16 \mu_{2}} \mid \begin{array}{c}
1,-k, \frac{\xi^{2}+2}{2} \\
\chi_{1}
\end{array}\right) d x,
\end{aligned}
$$

with $\chi_{1}$ previously defined.

Substituting (50) into (46), and then (40) and (46) into (39), a new analytical expression for the outage probability is derived as in (23).

\section{APPENDIX B}

\section{OUtAge Probability APPROXIMATION FOR $\rho=1$}

In the case of $\rho=1, \gamma_{1}$ and $\hat{\gamma}_{1}$ are fully-correlated exponentially distributed RVs. Hence, the overall SNR can be written as

$$
\gamma_{e q 1}=\frac{\gamma_{1} \gamma_{2}}{\gamma_{2}+\gamma_{1}}
$$

The well-known approximation, $\gamma_{e q_{1}} \min =\min \left(\gamma_{1}, \gamma_{2}\right)$, is used in order to obtain the outage probability for the system under the investigation in the form [25]

$$
\begin{aligned}
P_{\text {out }}^{\rho=1} & \approx \operatorname{Pr}\left(\min \left(\gamma_{1}, \gamma_{2}\right)<\gamma_{t h}\right) \\
& =1-\bar{F}_{\gamma_{1}}\left(\gamma_{t h}\right) \bar{F}_{\gamma_{2}}\left(\gamma_{t h}\right),
\end{aligned}
$$


where $\bar{F}_{\gamma_{2}}(x)$ is the CCDF of $\gamma_{2}$ defined by $\bar{F}_{\gamma_{2}}(x)=1-$ $F_{\gamma_{2}}(x)$, with the CDF $F_{\gamma_{2}}$ given by (20), while the CCDF of the exponentially distributed $\mathrm{RV} \gamma_{1}$ is

$$
\bar{F}_{\gamma_{1}}(x)=1-F_{\gamma_{1}}(x)=e^{-\frac{x}{\mu_{1}}},
$$

with $F_{\gamma_{1}}(x)$ being the $\mathrm{CDF}$ of $\gamma_{1}[31]$.

By substituting (20) and (53) into (52), the outage probability approximation for $\rho=1$ is derived as in (29).

\section{APPENDIX C \\ DERIVATION OF EQ. (31)}

The average BER of the system under the investigation can be derived by substituting (23) into (30)

$$
\begin{aligned}
& P_{b}=\frac{q^{p}}{2 \Gamma(p)} \int_{0}^{\infty} e^{-q \gamma} \gamma^{p-1}\left\{1-e^{-\frac{\gamma}{\mu_{1}}}\right. \\
& +\sum_{k=0}^{\infty} \sum_{n=0}^{k}\left(\begin{array}{l}
k \\
n
\end{array}\right) \frac{2^{\alpha+\beta-3} \xi^{2} \rho^{k}(1-\rho)^{n-k+1} \mu_{1}^{n-k}}{k !^{2} \pi \Gamma(\alpha) \Gamma(\beta)} \\
& \left.\times \gamma^{k-n} e^{-\frac{\gamma}{(1-\rho) \mu_{1}}} G_{3,7}^{6,2}\left(\frac{\alpha^{2} \beta^{2} \kappa^{2} \gamma}{16 \mu_{2}} \mid \begin{array}{c}
1,-k, \frac{\xi^{2}+2}{2} \\
\chi_{1}
\end{array}\right)\right\} d \gamma
\end{aligned}
$$

After separation of the integrals, (54) is rewritten as

$$
P_{b}=I_{3}-I_{4}+I_{5},
$$

where the integral $I_{3}$ is given and resolved by using [32, (3.351.3)] as

$$
I_{3}=\frac{q^{p}}{2 \Gamma(p)} \int_{0}^{\infty} e^{-q \gamma} \gamma^{p-1} d \gamma=\frac{1}{2},
$$

while the integral $I_{4}$ can be solved as [32, (3.351.3)]

$$
I_{4}=\frac{q^{p}}{2 \Gamma(p)} \int_{0}^{\infty} e^{-q \gamma} \gamma^{p-1} e^{-\frac{\gamma}{\mu_{1}}} d \gamma=\frac{1}{2}\left(\frac{1}{q \mu_{1}}+1\right)^{-p} .
$$

The integral $I_{5}$ has the form

$$
\begin{aligned}
I_{5}= & \frac{q^{p}}{2 \Gamma(p)} \sum_{k=0}^{\infty} \sum_{n=0}^{k}\left(\begin{array}{l}
k \\
n
\end{array}\right) \frac{2^{\alpha+\beta-3} \xi^{2} \rho^{k}}{k !^{2} \pi \Gamma(\alpha) \Gamma(\beta)} \\
& \times(1-\rho)^{n-k+1} \mu_{1}{ }^{n-k} \int_{0}^{\infty} \gamma^{k-n+p-1} e^{-\gamma\left(\frac{1}{(1-\rho) \mu_{1}}+q\right)} \\
& \times G_{3,7}^{6,2}\left(\frac{\alpha^{2} \beta^{2} \kappa^{2} \gamma}{16 \mu_{2}} \mid \begin{array}{c}
1,-k, \frac{\xi^{2}+2}{2} \\
\chi_{1}
\end{array}\right) d \gamma .
\end{aligned}
$$

Representing exponential function in terms of Meijer's $G$-function by [35, (01.03.26.0004.01)], this integral can be solved using $[35,(07.34 .21 .0011 .01)]$ as

$$
\begin{gathered}
I_{5}=\frac{q^{p}}{2 \Gamma(p)} \sum_{k=0}^{\infty} \sum_{n=0}^{k}\left(\begin{array}{l}
k \\
n
\end{array}\right) \frac{2^{\alpha+\beta-3} \xi^{2} \rho^{k}(1-\rho)^{p+1} \mu_{1}^{p}}{k !^{2} \pi \Gamma(\alpha) \Gamma(\beta)\left(1+q(1-\rho) \mu_{1}\right)^{k-n+p}} \\
\times G_{4,7}^{6,3}\left(\frac{\alpha^{2} \beta^{2} \kappa^{2}(1-\rho) \mu_{1}}{16 \mu_{2}\left(1+q(1-\rho) \mu_{1}\right)} \mid \begin{array}{c}
1,-k, 1-k+n-p, \frac{\xi^{2}+2}{2} \\
\chi_{1}
\end{array}\right) d \gamma .
\end{gathered}
$$

Finally, by substituting (56), (57), and (59) into (55), the average BER expression in (31) is derived.

\section{ACKNOWLEDGMENT}

The authors would like to thank Guest Editors and the reviewers for their valuable comments and discussions, which considerably improved the quality of the manuscript.

\section{REFERENCES}

[1] S. Arnon, J. Barry, G. Karagiannidis, R. Schober, and M. Uysal, Eds. Advanced Optical Wireless Communication Systems. New York, NY, USA: Cambridge Univ. Press, 2012.

[2] Z. Ghassemlooy, W. Popoola, and S. Rajbhandari, Optical Wireless Communications: System and Channel Modelling With MATLAB. Boca Raton, FL, USA: CRC Press, 2013.

[3] L. C. Andrews and R. N. Philips, Laser Beam Propagation Through Random Media, 2nd ed. Bellingham, WA, USA: SPIE, 2005.

[4] M. A. Al-Habash, L. C. Andrews, and R. N. Philips, "Mathematical model for the irradiance probability density function of a laser beam propagating through turbulent media," Opt. Eng., vol. 40, no. 8, pp. 1554-1562, Aug. 2001 .

[5] M. A. Khalighi and M. Uysal, "Survey on free space optical communication: A communication theory perspective," IEEE Commun. Surveys Tuts., vol. 16, no. 4, pp. 2231-2258, 4th Quart. 2014.

[6] M. Uysal, J. Li, and M. Yu, "Error rate performance analysis of coded free-space optical links over gamma-gamma atmospheric turbulence channels," IEEE Trans. Wireless Commun., vol. 5, no. 6, pp. 1229-1233, Jun. 2006.

[7] M. Safari and M. Uysal, "Relay-assisted free-space optical communication," IEEE Trans. Wireless Commun., vol. 7, no. 12, pp. 5441-5449, Dec. 2008.

[8] X. Tang, Z. Wang, Z. Xu, and Z. Ghassemlooy, "Multihop free-space optical communications over turbulence channels with pointing errors using heterodyne detection," J. Lightw. Technol., vol. 32, no. 15, pp. 2597-2604, Aug. 2014.

[9] A. Jurado-Navas, J. M. Garrido-Balsells, J. F. Paris, M. Castillo-Vazquez, and A. Puerta-Notario, "Further insights on Málaga distribution for atmospheric optical communications," in Proc. IWOW, Pisa, Italy, Oct. 2012, pp. 1-3.

[10] A. Jurado-Navas, J. M. Garrido-Balsells, J. F. Paris, M. Castillo-Vazquez, and A. Puerta-Notario, "General analytical expressions for the bit error rate of atmospheric optical communication systems," Opt. Lett., vol. 36, no. 20, pp. 4095-4097, Oct. 2011.

[11] S. Arnon, "Effects of atmospheric turbulence and building sway on optical wireless communication systems," Opt. Lett., vol. 28, no. 2, pp. 129-131, Jan. 2003.

[12] A. A. Farid and S. Hranilovic, "Outage capacity optimization for free space optical links with pointing errors," J. Lightw. Technol., vol. 25, no. 7, pp. 1702-1710, Jul. 2007.

[13] H. G. Sandalidis, T. A. Tsiftsis, and G. K. Karagiannidis, "Optical wireless communications with heterodyne detection over turbulence channels with pointing errors," J. Lightw. Technol., vol. 27, no. 20, pp. 4440-4445, Oct. 2009.

[14] H. G. Sandalidis, "Optimization models for misalignment fading mitigation in optical wireless links," IEEE Commun. Lett., vol. 12, no. 5, pp. 395-397, May 2008.

[15] A. A. Farid and S. Hranilovic, "Outage capacity for MISO intensitymodulated free-space optical links with misalignment," IEEE/OSA J. Opt. Commun. Netw., vol. 3, no. 10, pp. 780-789, Oct. 2011. 
[16] N. Mingbo, J. Cheng, and J. F. Holzman, "Error rate performance comparison of coherent and subcarrier intensity modulated optical wireless communications," IEEE/OSA J. Opt. Commun. Netw., vol. 5, no. 6, pp. 554-564, Jun. 2013.

[17] J. Li, J. Q. Liu, and D. P. Taylor, "Optical communication using subcarrier PSK intensity modulation through atmospheric turbulence channels," IEEE Trans. Commun., vol. 55, no. 8, pp. 1598-1606, Aug. 2007.

[18] W. O. Popoola and Z. Ghassemlooy "BPSK subcarrier intensity modulated free-space optical communications in atmospheric turbulence," $J$. Lightw. Technol., vol. 27, no. 8, pp. 967-973, Apr. 2009.

[19] Y. Yang, H. Hu, J. Xu, and G. Mao, "Relay technologies for WiMAX and LTE-advanced mobile systems," IEEE Commun. Mag., vol. 47, no. 10, pp. 100-105, Oct. 2009.

[20] E. Lee, J. Park, D. Han, and G. Yoon, "Performance analysis of the asymmetric dual-hop relay transmission with mixed RF/FSO links," IEEE Photon. Technol. Lett., vol. 23, no. 21, pp. 1642-1644, Nov. 2011

[21] I. S. Ansari, F. Yilmaz, and M.-S. Alouini, "On the performance of mixed RF/FSO dual-hop transmission systems," in Proc. IEEE 77th VTC Spring, Jun. 2013, pp. 1-5.

[22] I. S. Ansari, F. Yilmaz, and M.-S. Alouini, "Impact of pointing errors on the performance of mixed RF/FSO dual-hop transmission systems," IEEE Wireless Commun. Lett., vol. 2, no. 3, pp. 351-354, Jun. 2013.

[23] H. Samimi and M. Uysal, "End-to-end performance of mixed RF/FSO transmission systems," IEEE/OSA J. Opt. Commun. Netw., vol. 5, no. 11, pp. 1139-1144, Nov. 2013.

[24] I. S. Ansari, M.-S. Alouini, and F. Yilmaz, "On the performance of hybrid $\mathrm{RF}$ and RF/FSO fixed gain dual-hop transmission systems," in Proc. SIECPC, Apr. 2013, pp. 1-6.

[25] I. S. Ansari, F. Yilmaz, and M.-S. Alouini, "On the performance of mixed RF/FSO variable gain dual-hop transmission systems with pointing errors," in Proc. IEEE 78th VTC Fall, Sep. 2013, pp. 1-5.

[26] I. S. Ansari, F. Yilmaz, and M.-S. Alouini, "On the performance of hybrid RF and RF/FSO dual-hop transmission systems," in Proc. 2nd IWOW, Newcastle Upon Tyne, U.K., Oct. 2013, pp. 45-49.

[27] Z. Hadzi-Velkov, D. S. Michalopoulos, G. K. Karagiannidis, and R. Schober, "Dual-hop amplify-and-forward transmission with imperfect channel estimates at the relay," in Proc. IEEE ICC, Jun. 2012, pp. 4110-4115.

[28] Z. Hadzi-Velkov, D. S. Michalopoulos, G. K. Karagiannidis, and R. Schober, "On the effect of outdated channel estimation in variable gain relaying: Error performance and PAPR," IEEE Trans. Wireless Commun., vol. 12, no. 3, pp. 1084-1097, Mar. 2013.

[29] D. S. Michalopoulos, H. A. Suraweera, G. K. Karagiannidis, and R. Schober, "Amplify-and-forward relay selection with outdated channel estimates," IEEE Trans. Commun., vol. 60, no. 5, pp. 1278-1290, May 2012

[30] M. O. Hasna and M.-S. Alouini, "End-to-end performance of transmission systems with relays over Rayleigh-fading channels," IEEE Trans. Wireless Commun., vol. 2, no. 6, pp. 1126-1131, Nov. 2003.

[31] M. K. Simon and M.-S. Alouni, Digital Communication Over Fading Channels. 2nd ed. New York, NY, USA: Wiley, 2004.

[32] I. S. Gradshteyn and I. M. Ryzhik, Table of Integrals, Series, and Products, 6th ed. New York, NY, USA: Academic, 2000.

[33] H. E. Nistazakis, T. A. Tsiftsis, and G. S. Tombras, "Performance analysis of free-space optical communication systems over atmospheric turbulence channels," IET Commun., vol. 3, no. 8, pp. 1402-1409, Aug. 2009.

[34] V. S. Adamchik and O. I. Marichev, "The algorithm for calculating integrals of hypergeometric type function and its realization in reduce system," in Proc. Int. Conf. Symbolic Algebraic Comput., Tokyo, Japan, 1990, pp. 212-224.

[35] The Wolfarm Functions Site, 2008. [Online]. Available: http:/functions. wolfarm.com

[36] I. S. Ansari, S. Al-Ahmadi, F. Yilmaz, M.-S. Alouini, and H. Yanikomeroglu, "A new formula for the BER of binary modulations with dual-branch selection over generalized-K composite fading channels," IEEE Trans. Commun., vol. 59, no. 10, pp. 2654-2658, Oct. 2011

[37] A. H. Wojnar, "Unknown bounds on performance in Nakagami channels," IEEE Trans. Commun., vol. 34, no. 1, pp. 22-24, Jan. 1986.

[38] Y. R. Zheng and C. Xiao, "Simulation models with correct statistical properties for Rayleigh fading channels," IEEE Trans. Commun., vol. 51, no. 6, pp. 920-928, Jun. 2003 .

[39] G. Marsaglia and W. W. Tsang, "A simple method for generating gamma variables," ACM Trans. Math. Softw., vol. 26, no. 3, pp. 363-372, Jan. 2000 .
[40] D. Kundu and R. D. Gupta, "A convenient way of generating gamma random variables using generalized exponential distribution," Comput. Stat. Data Anal., vol. 51, no. 6, pp. 2796-2802, Mar. 2007.

[41] D. A. Luong, T. C. Thang, and A. T. Pham, "Effect of avalanche photodiode and thermal noises on the performance of binary phaseshift keying-subcarrier-intensity modulation/free-space optical systems over turbulence channels," IET Commun., vol. 7, no. 8, pp. 738-744, May 2013.

[42] B. He and R. Schober, "Bit-interleaved coded modulation for hybrid RF/FSO systems," IEEE Trans. Commun., vol. 57, no. 12, pp. 3753-3763, Dec. 2009.

[43] N. D. Chatzidiamantis, G. K. Karagiannidis, E. E. Kriezis, and M. Matthaiou, "Diversity combining in hybrid RF/FSO systems with PSK modulation," in Proc. IEEE ICC, pp. 1-6, Jun. 2011.

[44] K. P. Peppas, G. C. Alexandropoulos, C. K. Datsikas, and F. I. Lazarakis, "Multivariate gamma-gamma distribution with exponential correlation and its applications in radio frequency and optical wireless communications," IET Microw. Antennas Propag., vol. 5, no. 3, pp. 364-371, Feb. 2011.

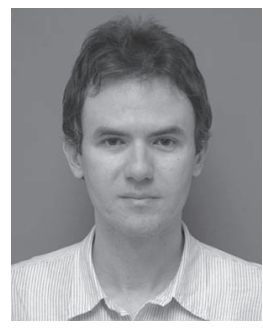

Goran T. Djordjevic (M'01) was born in Nis, Serbia. He received the B.S., M.S., and Ph.D. degrees in electrical engineering from the Faculty of Electronic Engineering, University of Nis, Serbia, in 1996, 1999, and 2005, respectively. Currently, he is an Associate Professor at the Department of Telecommunications, Faculty of Electronic Engineering, University of Nis, Serbia. He teaches courses in communication theory, modeling and simulation of communication systems, information theory and satellite communications.

His area of interest is communication theory and applications in satellite, wireless and optical communication systems. His current research interests include application of different modulation formats and error control codes in free-space optical systems, as well as modeling and simulation of fading channels.

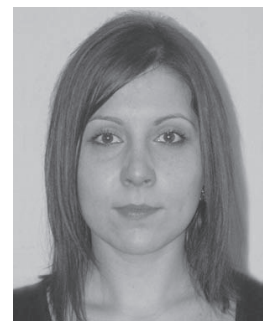

Milica I. Petkovic (S'12) was born in Knjazevac, Serbia, in 1986. She received the M.Sc. degree in electrical engineering from the Faculty of Electronic Engineering, University of Nis, Nis, Serbia, in 2010, and is enrolled in Ph.D studies with the same faculty. Her research interests include communication theory, wireless and optical communication systems, application of different modulation techniques and modeling of fading channels. She has been working towards her Ph.D degree in the field of free-space optical systems.

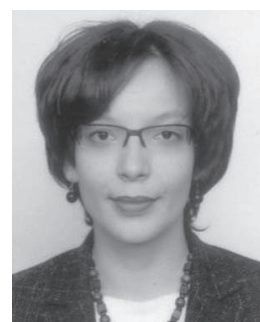

Aleksandra M. Cvetkovic (S'12) was born in Nis, Serbia. She received the B.S., M.S., and Ph.D degrees in electrical engineering from the Faculty of Electronic Engineering, University of Nis, Nis, Serbia, in 2001, 2007, and 2013, respectively. She is currently working with the Faculty of Electronic Engineering, Nis, Serbia, as an Assistant. Her research interests include wireless communication theory, cooperative communication and free-space optical systems. Other areas of research include statistical characterization and modeling of fading channels and performance analysis of multi user wireless systems subject to interference. She has published several papers on the above subjects. 


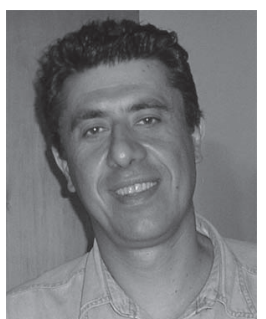

George K. Karagiannidis (M'96-SM'03-F'14)

was born in Pithagorion, Samos Island, Greece. He received the University Diploma (5 years) and Ph.D. degree in electrical and computer engineering from the University of Patras, Patras, Greece, in 1987 and 1999, respectively. From 2000 to 2004, he was a Senior Researcher at the Institute for Space Applications and Remote Sensing, National Observatory of Athens, Greece. In June 2004, he joined the faculty of Aristotle University of Thessaloniki, Greece, where he is currently Professor in the Electrical \& Computer Engineering Department and Director of the Digital Telecommunications Systems and Networks Laboratory. In January 2014, he joined Khalifa University, UAE, where he is currently a Professor in the Electrical \& Computer Engineering Department and Coordinator of the ICT Cluster.

His research interests are in the broad area of digital communications systems with emphasis on communications theory, energy efficient MIMO and cooperative communications, satellite communications, cognitive radio, localization, smart grid and optical wireless communications.

$\mathrm{He}$ is the author or co-author of more than 250 technical papers published in scientific journals and presented at international conferences. He is also author of the Greek edition of the book Telecommunications Systems and co-author of the book Advanced Optical Wireless Communications Systems, (Cambridge Publications, 2012). He is co-recipient of the Best Paper Award of the Wireless Communications Symposium (WCS) in the IEEE International Conference on Communications (ICC'07), Glasgow, U.K., June 2007.

Dr. Karagiannidis has been a member of Technical Program Committees for several IEEE conferences such as ICC, GLOBECOM, and VTC. In the past, he was Editor for Fading Channels and Diversity of the IEEE TRANSACTIONS ON COMMUNICATIONS, Senior Editor of IEEE COMMUNICATIONS LETTERS and Editor of the EURASIP Journal of Wireless Communications \& Networks. He was Lead Guest Editor of the special issue on "Optical Wireless Communications" of the IEEE JOURNAL ON SELECTED AREAS IN COMMUNICATIONS and Guest Editor of the special issue on "Large-scale multiple antenna wireless systems."

Since January 2012, he is the Editor-in Chief of IEEE COMMUNICATIONS LETTERS. 